\title{
PERCEPÇÕES DE PACIENTES AMPUTADOS HOSPITALIZADOS: UMA EXPLORAÇÃO QUALITATIVA DO PERÍODO PÓS-OPERATÓRIO
}

\author{
Ruy Luiz Lorenzetti Branco ${ }^{1}$ \\ Kadine Priscila Bender dos Santos ${ }^{2}$ \\ Soraia Cristina Tonon da $\mathrm{Luz}^{3}$ \\ Gesilani Júlia da Silva Honório 4 \\ Paulo Adão de Medeiros ${ }^{5}$ \\ Recebido em: 15 abr. 2018 \\ Aceito em: 22 mar. 2019
}

RESUMO: Objetivo: Conhecer as percepções de pacientes amputados no período pósoperatório imediato frente às Diretrizes de Atenção à Pessoa Amputada. Métodologia: Foram realizadas 10 entrevistas com sujeitos em pós-operatório imediato de amputação de membros inferiores em um hospital público. As entrevistas foram áudio-gravadas, transcritas e analisadas pelo software Qualitative Solutions Research (QSR) Nvivo versão 11. Resultados: A entrevista intitulada "Conversa no Leito", abordou cinco temas geradores, que revelaram por meio de suas falas, aspectos desde a reabilitação à atuação da equipe multidisciplinar no ambiente hospitalar. Os pacientes manifestaram tristeza pela perda do membro, e a família foi a principal responsável pelo empoderamento no ambiente hospitalar. Conclusão: As percepções dos pacientes evidenciaram a aplicabilidade das diretrizes no ambiente hospitalar. As diretrizes são instrumentos norteadores e servem de guia para a equipe multidisciplinar necessitando ser mais utilizada, otimizando o processo de reabilitação.

Palavras-chave: Fisioterapia; Amputação; Serviço Hospitalar de Fisioterapia; Educação em saúde; Cuidados Pós-Operatórios.

\section{PERCEPTIONS OF PATIENTS HOSPITALIZED AMPUTEES: A QUALITATIVE}

\section{EXPLORATION OF THE POSTOPERATIVE PERIOD}

ABSTRACT: Objective: To know the perceptions of amputees in the immediate postoperative period in front of the Guidelines for Attention to Amputees. METHODS: Ten interviews were conducted with subjects in the immediate postoperative period of lower limb amputation in a public hospital. The interviews were audio-taped, transcribed and analyzed by Qualitative Solutions Research (QSR) software. Results:

\footnotetext{
${ }^{1}$ Ruy Luiz Lorenzetti Branco, fisioterapeuta, mestrando do Programa de Pós-graduação em Fisioterapia Universidade do Estado de Santa Catarina - UDESC, Florianópolis, SC, Brasil. Endereço para correspondência: Ruy Luiz Lorenzetti Branco - Laboratório de Biomecânica/Centro de Ciências da Saúde e do Esporte/CEFID, Universidade do Estado de Santa Catarina/UDESC, Rua Pascoal Simone, 385 Coqueiros - Florianópolis - SC. CEP: 88080-350 - Fone 55 (48) 3321-8670 - ruy.I.branco@hotmail.com.

${ }^{2}$ Kadine Priscila Bender dos Santos, mestre em educação, Universidade do vale do Itajaí - UNIVALI e em Fisioterapia, Universidade do Estado de Santa Catarina - UDESC, Florianópolis, SC, Brasil.

${ }^{3}$ Soraia Cristina Tonon da Luz, docente do curso de Fisioterapia, Universidade do Estado de Santa Catarina - UDESC, Florianópolis, SC, Brasil.

${ }^{4}$ Gesilani Júlia da Silva Honório, docente do curso de Fisioterapia, Universidade do Estado de Santa Catarina - UDESC, Florianópolis, SC, Brasil.

${ }^{5}$ Paulo Adão de Medeiros, mestre em Ciências do Movimento Humano, Universidade do Estado de Santa Catarina - UDESC, Florianópolis, SC, Brasil.
} 
The interview entitled "Conversation in the Bed", addressed five generative themes, which revealed, through their lines, aspects from rehabilitation to the performance of the multidisciplinary team in the hospital environment. Patients expressed sadness over the limb loss, and the family was primarily responsible for empowerment in the hospital setting. Conclusion: Patients' perceptions evidenced the applicability of the guidelines in the hospital environment. The guidelines are guiding instruments and serve as a guide for the multidisciplinary team needing to be more used, optimizing the rehabilitation process.

Keywords: Physical Therapy Specialty; Amputation; Physical Therapy Department, Hospital; Health education; Postoperative Care.

\section{INTRODUÇÃO}

No Brasil, o número de amputados vem aumentando significativamente (BRASIL, 2012; HAWKINS et al., 2013), Segundo o Instituto Brasileiro de Geografia Estatística (IBGE) (CENSO DEMOGRÁFICO, 2010), das 13,2 milhões de pessoas que declararam apresentar algum tipo de deficiência motora no Brasil, 470 mil sofreram amputações, sendo o diabetes mellitus (DM) é uma das principais etiologias que se relaciona à essas amputações (SBD, 2015), seguido dos traumas, doenças oncológicas, infecciosas e condições congênitas (QUILICl et al., 2016). Aproximadamente 1 milhão de pessoas com DM apresenta amputação de parte da perna anualmente, e supõe-se que a cada 20 segundos um membro inferior é amputado no mundo resultante desta doença (IWGDF, 2015).

Devido a essa demanda importante, o Brasil dispõe de diretrizes de atenção à saúde da pessoa amputada (BRASIL, 2013), orientando os profissionais desde os cuidados no ambiente hospitalar, que compreendem a fase pré-cirúrgica, cirúrgica e pós-cirúrgica. Estes cuidados abarcam desde o preparo da pessoa ao processo cirúrgico, como a escolha do nível de amputação, avaliação dos aspectos cognitivos, assim como preparação do paciente para o momento da notícia da amputação.

Na fase pós-cirúrgica imediata, estas diretrizes orientam os a equipe multidisciplinar o cuidado da pessoa no ambiente hospitalar logo após a amputação, esses cuidados se remetem a transferências de decúbito, deslocamentos com o auxílio de dispositivos auxiliares, treino de marcha, orientação sobre o correto posicionamento do segmento para evitar deformidades, controle do edema e modelagem do coto, exercícios de fortalecimento muscular e dessensibilização da região amputada.

As diretrizes orientam ainda o estímulo ao emponderamento do paciente, necessário para a efetiva alta hospitalar, e reforça a necessidade de encaminhamentos nos diferentes pontos de atenção da rede de cuidados à pessoa com deficiência, para a continuidade do processo de reabilitação (BRASIL, 2013).

A literatura científica é escassa em relação ao conhecimento das percepções dos pacientes amputados no período imediato a amputação, ainda em ambiente hospitalar. Parte-se da premissa que informações obtidas através do depoimento dos principais envolvidos, as pessoas amputadas, auxiliam a entender o processo de atendimento 
prestado na prática para melhorar a efetividade de diretrizes e protocolos específicos de acolhimento e tratamento no ambiente hospitalar. Falhas nas orientações fornecidas aos pacientes amputados hospitalizados podem repercutir em desfechos negativos para 0 processo de reabilitação pós-alta hospitalar. Orientações e informações necessárias, como por exemplo, sobre a protetização, estão contempladas nas diretrizes e servem como questões norteadoras da comunicação pela equipe interdisciplinar, e principalmente, entre profissional e paciente, no entanto, a rede de atenção deve funcionar para o processo de reabilitação ser efetivo.

Nesse contexto, o objetivo do estudo foi conhecer as percepções dos pacientes amputados hospitalizados no período pós-operatório imediato frente às Diretrizes de Atenção à Pessoa Amputada.

\section{MATERIAIS E MÉTODOS}

Trata-se de um estudo exploratório descritivo (GASKEL; BAUER, 2002), com abordagem qualitativa, aprovado pelos comitês de ética: da UDESC (CAAE $\mathrm{n}^{\circ}$ 33009514.9.3001.0112) e do Hospital ( $n$-33009514.9.3001.0112). Optou-se por um estudo exploratório por consistir em uma maior proximidade com o universo do objeto de estudo pesquisado. Esse tipo de estudo visa, através dos métodos e dos critérios, oferecer informações e orientar a formulação das hipóteses do estudo. E descritivo por se realizar um estudo detalhado, com levantamento de informações através das técnicas de coleta, sendo que o pesquisador age apenas como observador, sem assumir qualquer tipo de interferência ao objeto de estudo. Este, por sua vez, deve ser analisado no seu ambiente natural, para que uma maior veracidade do registro seja alcançada (GASKEL; BAUER, 2002).

O referencial teórico do estudo foi baseado nas Diretrizes de Atenção à Pessoa Amputada (BRASIL, 2013), que tem como um dos objetivos fornecer às equipes multiprofissionais o cuidado à saúde de pessoas com amputação de membros, desde o ambiente hospitalar até o ambulatorial. Para o desenvolvimento da pesquisa foram respeitados os princípios éticos estabelecidos pela Resolução n.466/12 do Conselho Nacional de Saúde do Ministério da Saúde. Todos os participantes do estudo assinaram o Termo de Esclarecimento Livre e Esclarecido (TCLE).

A coleta de dados foi realizada no setor de ortopedia de um hospital referência em cirurgia de amputação na cidade de São José. Para a participação na pesquisa, os indivíduos deveriam apresentar como critérios de inclusão idade acima de dezoito anos e estar em período pós-operatório imediato de amputação de membros inferiores, como critério de exclusão, não terem assinado o TCLE, apresentarem déficit cognitivo ou sobe efeito de algum medicamento que impossibilite a comunicação com o pesquisador.

O momento de finalizar as coletas dos dados foi por meio da saturação das falas que frequentemente é empregada nas investigações qualitativas em diferentes áreas no 
campo da saúde, entre outras. É usada para estabelecer ou fechar o tamanho final de uma amostra em estudo, interrompendo a captação de novos componentes (FONTANELLA et al. 2008). Esta pesquisa está vinculada às ações do projeto de extensão Reabilitação Multidisciplinar em Amputados (SANTOS; LUZ, 2015) do Centro de Ciências da Saúde e do Esporte (CEFID) da Universidade do Estado de Santa Catarina (UDESC).

\section{PROCEDIMENTO E COLETA DE DADOS}

Dos 62 sujeitos com amputação de membros inferiores notificados no período deste estudo, dez participaram da pesquisa devido a alguns sujeitos não atenderem aos critérios de inclusão ou recusarem participar do estudo. Para que esta coleta de dados fosse possível, anteriormente fora elaborada metodologia de notificação hospitalar do paciente amputado via projeto de extensão a partir da equipe multiprofissional do Hospital.

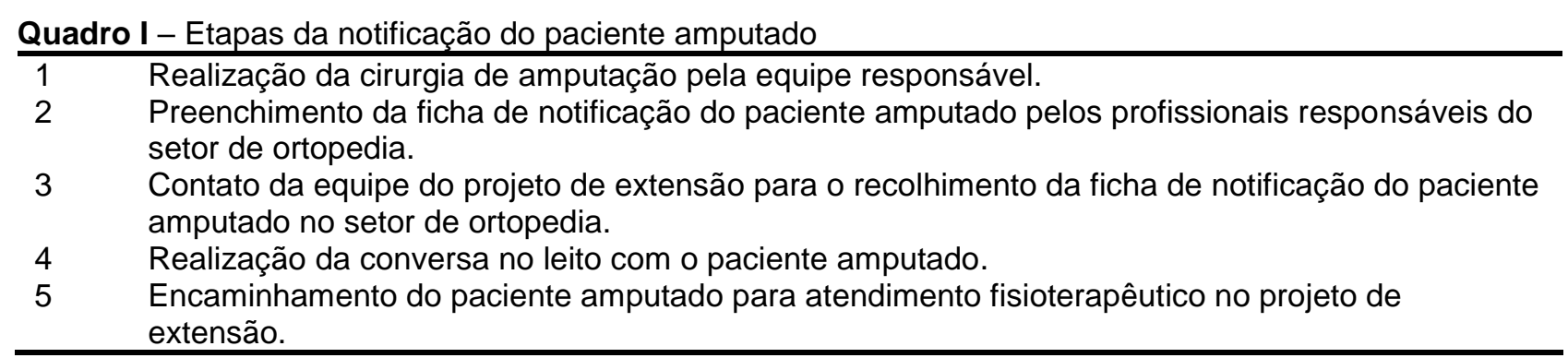

Fonte: BRANCO et al., 2017

Além da confecção da ficha pertencente às etapas da notificação do paciente amputado contendo dados principais do paciente e o nome do profissional que notificou, ela constituiu uma ferramenta de comunicação entre o pesquisador e os profissionais de saúde que trabalhavam com o paciente amputado no período pós-operatório. 0 preenchimento formal do documento foi realizado através de pasta dispostas no setor hospitalar, além disso, via mensagem telefônica para o pesquisador que se deslocava ao hospital para a realização da conversa com o paciente.

Durante o período de maio de 2015 a fevereiro de 2017, a dinâmica compreendeu a visita do pesquisador frente estas notificações para que pudesse ocorrer a coleta de dados. Realizou a partir de entrevista semiestruturada intitulada: "conversa no leito", sendo elaborada pelos pesquisadores, com duração média de 40 a 50 minutos (BRANCO et al., 2017). A conversa no leito abarcou cinco temas geradores, extraídos do que preconiza as Diretrizes de Atenção à Pessoa Amputada (BRASIL, 2013): Reabilitação; Resiliência; Amputação; Incertezas; e Orientação (Quadro I). 
Quadro II - Temas geradores da conversa no leito baseados na Diretriz de Atenção à Saúde da Pessoa Amputada.

\begin{tabular}{ll}
\hline $\begin{array}{l}\text { Temas } \\
\text { geradores }\end{array}$ & Questões norteadoras \\
& \\
\hline Reabilitação & - O que você espera do processo de reabilitação? \\
& - Quais profissionais vieram te informar sobre a existência do processo de reabilitação? \\
\hline Resiliência & - O que está sendo mais difícil no período de permanência no hospital? \\
& - Você está recebendo ajuda de alguém nessa fase de permanência no hosital? \\
\hline Amputação & - Para você, o que significa ter uma amputação? \\
& - Você conhece alguém que sofreu uma amputação? \\
\hline Incertezas & - Alguém já te falou sobre reabilitação para pessoas amputadas? \\
& - Algum profissional do hospital te informou sobre a existência de prótese? \\
Orientação & - Você recebeu alguma orientação após a amputação? \\
& - Você acha importante receber orientação de algum profissional logo após a \\
& amputação? Por quê?
\end{tabular}

Fonte: BRANCO et al., 2017

\section{PROCEDIMENTOS DE ANÁLISE DE DADOS}

As entrevistas foram áudio- gravadas e, após, realizou-se a transcrição fidedigna de seu conteúdo na íntegra, o qual foi transferido para o software Qualitative Solutions Research (QSR) Nvivo versão 11(QRS INTERNACIONAL PTY LTD, 2017), a fim de identificar nas falas dos sujeitos a frequência do conteúdo emitido, sendo as falas dispostas por tema gerador. Optou-se pelo método de codificação manual, ferramenta do software, fazendo-se a codificação de unidades de texto em categorias, ou seja, cada resposta/fala dos entrevistados foram categorizadas por temas geradores baseados no referido referencial teórico.

A análise dos dados resultou nas seguintes palavras-chave: fisioterapeuta, reabilitação, prótese, família, Deus, amputar, perder, dúvidas, conversar, médico e enfermeira. Os resultados são apresentados por essas palavras-chave, com identificação do tema gerador, assim como respectivas falas dos entrevistados.

\section{CARACTERIZAÇÃO DOS SUJEITOS}

As características das dez pessoas entrevistadas foram: uma pessoa do sexo feminino com 19 anos, com amputação unilateral de etiologia traumática. $\mathrm{N}$ ove homens com idade entre 23 e 67 anos, com amputação unilateral de etiologia vascular e um bilateral de etiologia traumática. 


\begin{tabular}{llllll}
\multicolumn{6}{l}{ Quadro III - Caracterização dos participantes. } \\
\hline IDENTIFICAÇÃO & IDADE & SEXO & ETIOLOGIA & NíVEL & ACOMETIMENTO \\
\hline E1 & 19 & Feminino & Traumática & Transtibial & Unilateral \\
E2 & 23 & Masculino & Traumática & Transfemural & Unilateral \\
E3 & 67 & Masculino & Vascular & Transfemural & Unilateral \\
E4 & 38 & Masculino & Traumática & Transfemural & Bilateral \\
E5 & 25 & Masculino & Vascular & Transtibial & Unilateral \\
E6 & 46 & Masculino & Traumática & Transtibial & Unilateral \\
E7 & 32 & Masculino & Traumática & Transfemural & Unilateral \\
E8 & 31 & Masculino & Vascular & Transtibial & Unilateral \\
E9 & 32 & Masculino & Traumática & Transfemural & Unilateral \\
E10 & 28 & Masculino & Vascular & Transfemural & Unilateral \\
\hline
\end{tabular}

Fonte: Elaborado pelos autores.

\section{RESULTADOS E DISCUSSÃO}

\section{REABILITAÇÃO}

Foi investigado nesse tema gerador se a reabilitação da pessoa amputada iniciou no ambiente hospitalar e se recebeu informações sobre este processo. Investigou-se, também, as expectativas da pessoa amputada sobre a reabilitação.

A seguir, algumas falas exploram os resultados encontrados:

"a fisioterapeuta falou, falou dos exercícios que eu tinha que fazer" (E3, homem, 67 anos)

"a fisioterapeuta disse que eu vou ter que fazer fisioterapia para pessoa que amputou que é um pouco diferente assim".

(E1, mulher, 19 anos)

"foi a própria fisioterapeuta que teve ontem aqui, ela teve dando umas dicas e disse que assim que tivesse alta ia ter um encaminhamento para a minha cidade [...] então vão me encaminhar para um centro de reabilitação para fazer a Fisioterapia".

(E5, homem, 25 anos)

"as fisioterapeutas vieram aqui conversar, o pessoal da faculdade veio me ensinar uns exercícios, disseram que eu posso fazer fisioterapia lá duas vezes por semana".

(E10, 28 anos, masculino)

As diretrizes brasileiras preconizam que na fase pós-cirúrgica imediata à equipe multidisciplinar é a responsável pelos cuidados ao paciente no ambiente hospitalar logo após a amputação. Expõe que no pós-operatório imediato, anterior ao treino de marcha, toda pessoa amputada deve ser orientada como realizar suas transferências de postura e deslocamentos, orientações quanto ao posicionamento, prevenção de deformidades, 
controle de edema e principalmente, modelagem do coto para uma futura protetização. Ainda trazem que em relação à fisioterapia, um programa de exercícios com a finalidade de corrigir ou prevenir deformidades, aumentar força, mobilidade e equilíbrio, deve ser estabelecido (BRASIL, 2013).

Como revelado nas falas, às diretrizes destacam que no processo de amputação, apesar de se tratar de procedimento realizado pelos níveis de maior complexidade, as pessoas devem ser atendidas e acompanhadas pelos profissionais da atenção básica e direcionadas, se necessário, aos demais serviços da rede de atenção (PETERMANN et al., 2015).

Além disso, a equipe multidisciplinar precisa se sensibilizar das reais necessidades das pessoas submetidas à amputação, para que as orientações fornecidas sejam repercutidas positivamente a curto, médio e longo prazo e assim, evitando problemas de saúde relacionados com a amputação. Conhecer os diversos serviços de reabilitação e seus fluxos é vital, uma vez que no estado, a partir da deliberação 502/CIB/ 14 de março de 2015 (GOVERNO DE SANTA CATARINA, 2015), houve a descentralização dos serviços de reabilitação, ficando sob a responsabilidade dos municípios, sendo as pessoas amputadas remanejadas para os cuidados da atenção básica. Neste contexto, a equipe multidisciplinar deve realizar a atenção integral à saúde da pessoa amputada nos três níveis de atenção. Nesta circunstãncia, prevê-se o pré e pós-operatório imediato e a reabilitação pré e pós- protetização (BRASIL, 2013).

\section{RESILIÊNCIA}

O tema gerador abordou se, no ambiente hospitalar, houve estímulo de enfrentamento e de tomada de decisões relacionadas ao autocuidado saúde.

A seguir, destacam-se algumas emissões:

"olha cara, não tem preço, a família do teu lado te apoiando, família é o que te trás força". (E4, homem, 38 anos)

"eu me desanimei bastante, e pensei, porque aconteceu comigo, porque eu, mas Deus sabe de todas as coisas, alguma proposta, alguma coisa ele tem para minha vida para ter acontecido isso, Deus da à cruz para cada um, mas eu consigo carregar, e eu agarrei isso pra mim, eu estou carregando a minha né, estou conseguindo levar esse fardo, eu sei que eu não vou ser mais a mesma pessoa".

(E4, homem, 38 anos)

As diretrizes brasileiras (BRASIL, 2013) indicam que é fundamental ter uma equipe capacitada para lidar com o assunto frente à pessoa amputada e sua família e ainda, se possível, deve-se compreender que as inseguranças do paciente e da família são muitas, entre elas, a possibilidade de cura com esse tratamento, potencial de adaptação, autonomia futura e qualidade de vida. Nas falas das pessoas amputadas sobre o tema gerador resiliência, ficou claro o sentimento de tristeza e desânimo vivenciados logo após a amputação, porém relataram a importância da família no apoio à busca pela recuperação. 
O processo de empoderamento possibilita ao paciente amputado o processo educativo, estimulando o desenvolvimento de novas habilidades, conhecimentos, atitudes e o autoconhecimento, fazendo-se necessário para a tomada de decisão acerca de sua saúde (SAMUEL et al., 2017).

A diretriz norte americana Clinical Practice Guideline for Rehabilitation of Lower Limb Amputation (THE UNITED STATES OF AMERICA DEPARTMENT OF VETERANS AFFAIRS, 2007) expõe que a família tem um papel fundamental no empoderamento desses indivíduos, encorajando principalmente a sua recuperação. Ela será a responsável por incentivar questões como a adoção de um estilo de vida mais saudável, realização de exercícios, ingestão adequada de alimentos e, em alguns casos, até a cessação do tabagismo. As diretrizes brasileiras (BRASIL, 2013) complementam que a troca de experiências e o contato com outras pessoas que sofreram uma amputação auxiliam no esclarecimento de dúvidas e na superação de dificuldades.

Lange e Heuft (2001) pontuam que a indicação da intervenção de uma equipe de psicologia deve ser traçada nas necessidades individuais de cada paciente. Os autores ainda destacam que o trabalho é complexo ao abranger questões sociais, psíquicas e biológicas, necessitando da cooperação dos diversos especialistas envolvidos no processo cirúrgico. A atuação da psicologia na equipe interdisciplinar é de extrema importância na atuação com o paciente, sua rede de apoio e com a equipe de saúde, visando à minimização de sofrimentos decorrentes do processo de hospitalização, do adoecimento e do procedimento cirúrgico, proporcionando desenvolvimento da autonomia e corresponsabilização no processo de tratamento.

\section{AMPUTAÇÃO}

Este tema gerador teve como objetivo compreender como a pessoa reagiu à nova situação que está atribuída ao ato da amputação e a perda do membro, além de verificar a experiência da nova fase de vida no ambiente hospitalar.

As falas a seguir destacam as alterações emocionais que se envolvem no processo da amputação:

"significa perder uma parte de mim, para mim no começo foi muito triste, não conseguia olhar para ninguém, falar com ninguém, queria morrer, mas agora já estou bem melhor, a psicóloga está me ajudando bastante".

(E1, mulher, 19 anos)

"cara, vou dizer pra ti que seja uma tristeza, é uma parte do teu corpo que você perdeu, ninguém quer perder uma parte do corpo, uma amputação se torna uma coisa tão triste, mas tomara que essa coisa triste se torne meu crescimento".

(E7, homem, 32 anos)

"ahh, uma amputação significa um terror, porque a gente perde o membro da gente, perder um membro é um terror".

(E6, homem, 46 anos) 
"ah, como é que é, fiquei muito tempo internado, indo e voltando né, lutando contra a bactéria, aí o médico falou pra mim, não tem mais o que fazer, vamos ter que amputar, aí na hora eu neguei, depois eu tive que aceitar, ou era a perna ou era minha vida".

(E8, homem, 31 anos)

"ah, como é que é, fiquei muito tempo internado, indo e voltando né, lutando contra a bactéria, aí o médico falou pra mim, não tem mais o que fazer, vamos ter que amputar, aí na hora eu neguei, depois eu tive que aceitar, ou era a perna ou era a vida.

(E3, homem, 67anos)

Nos casos de cirurgias eletivas de amputação, a pessoa amputada necessita de uma preparação psicológica prévia. Esta preparação reforça a habilidade do mesmo em lidar com o processo operatório e aceitar a prótese na fase de reabilitação. Em casos de cirurgias eletivas, o momento da notícia da amputação deve ser realizado junto à equipe multidisciplinar, que deve estar preparada para informar ao paciente e seus familiares sobre esta tomada de decisão clínica diante de sua condição de saúde. Ainda, o guideline norte americano trás que e os membros da família e cuidadores devem ser parte da equipe de reabilitação, auxiliando no aspecto afetivo e emocional do paciente (THE UNITED STATES OF AMERICA DEPARTMENT OF VETERANS AFFAIRS, 2007).

$O$ acolhimento da equipe multiprofissional possui o intuito de informar e apresentar as possibilidades de retorno ao trabalho e profissional. A pessoa amputada em muitos casos necessita de apoio psicológico prévio antes do processo operatório, incentivando a aceitação do início do processo de reabilitação (BRASIL, 2013). Esse processo ainda irá diminuir seus medos, ansiedades e angústias, melhorando a recuperação e contribuindo para a alta hospitalar (BROOMHEAD et al., 2006). Corroborando com as diretrizes, no caso da presente pesquisa, indicado pela fala dos participantes, o psicólogo atrelado à equipe multidisciplinar também foi um dos responsáveis por orientar os pacientes em relação à amputação, amenizando muitas vezes as suas dúvidas, medos, ansiedades e anseios.

\section{INCERTEZAS}

O objetivo foi compreender as dúvidas das pessoas amputadas em relação ao processo de reabilitação no ambiente hospitalar e pós-alta.

As falas enunciaram:

\footnotetext{
"eu na verdade pra falar não tenho muitas dúvidas, não vou mentir né, não tenho muitas dúvidas".

(E2, homem, 24 anos).

“agora, agora não tenho, no decorrer do tratamento vai criando dúvidas e a gente vai perguntando".

(E9, homem, 32 anos).

“as minhas dúvidas sobre o amanhã ou sobre hoje?".

(E2, homem, 23 anos).
}

As falas das pessoas amputadas em relação ao tema gerador incertezas trouxeram 
que no período pós-operatório imediato à cirurgia de amputação, os pacientes não tiveram dúvidas, entretanto, destacam que, no decorrer da hospitalização e do processo de reabilitação, essas dúvidas poderão surgir. O período de hospitalização mostra-se assustador para alguns indivíduos, que muitas vezes passam dias ou meses internados, saindo de sua rotina e vivenciando situações de medo, tensão e angústias (VARGAS et al., 2014).

No ambiente hospitalar, e principalmente no pós-operatório imediato, as dúvidas, incertezas e inseguranças do paciente e da família normalmente são muitas, sendo importante orientar e esclarecer possibilidades de recuperação plena através de tratamentos, quanto ao seu potencial de adaptação, de autonomia futura e de qualidade de vida (THE UNITED STATES OF AMERICA DEPARTMENT OF VETERANS AFFAIRS, 2007).

As diretrizes apontam que é de extrema importância o papel da Atenção Básica ofertar orientações para o esclarecimento de dúvidas sobre o cuidado da pessoa amputada antes e após amputação. Os profissionais desse nível de atenção são responsáveis, por exemplo, por monitorar e proporcionar cuidado aos usuários que apresentam diagnóstico de doenças que podem ter como resultado a amputação, além de oferecer o cuidado integral a esse usuário, que não deve ser considerado apenas como uma pessoa amputada (BRASIL, 2013).

\section{ORIENTAÇÃO}

O tema gerador teve como intuito saber se as pessoas amputadas receberam orientação pós-amputação imediata no leito e se achavam pertinente receber essas orientações dos profissionais de saúde no ambiente hospitalar.

As seguintes falas revelaram:

"a enfermeira, eu perguntei pra ela ontem se tinha como eu colocar uma prótese e ela disse que tem".

(E7, homem, 32 anos)

"veio uma fisioterapeuta pela manhã, ela veio pela equipe do médico, ela disse mais ou menos como eu deveria ficar aqui, e depois o pessoal iria vir".

(E2, homem, 23 anos)

As diretrizes recomendam que a reabilitação da pessoa amputada contemple a participação de toda a equipe multiprofissional, que pode ser composta, por médicos, enfermeiros, fisioterapeutas, terapeutas ocupacionais e psicólogos. O projeto terapêutico e os objetivos traçados para o paciente devem ser pactuados dentro da equipe multiprofissional, objetivando garantir uma atenção integral e evitando a existência de condutas conflituosas (BRASIL, 2013).

Como exposto nas falas, os pacientes questionaram mais aos enfermeiros e aos médicos, no entanto, quem forneceu mais orientações foram os fisioterapeutas, expresso 
no tema gerador reabilitação, isso pode estar atrelado à função exercida pelo fisioterapeuta no processo de protetização.

Diante da fala do paciente, não há como neste estudo aferir que este pode apresentar incertezas quanto aos cuidados da pessoa amputada, percebido na fala, a saber:

"meu médico falou que tem só que ele falou que não ia dar informação errada que pode ter como eu usar prótese".

(E10, masculino, 28 anos)

As Diretrizes de Atenção à Pessoa Amputada (BRASIL, 2013), no ambiente hospitalar, foram, de certa forma, contempladas neste ambiente hospitalar, pois se identificou através das percepções relatadas pelos sujeitos do estudo, que as orientações quanto ao processo de reabilitação foram fornecidas pelos fisioterapeutas.

Corroborando com as diretrizes brasileiras, o guideline inglês intitulado Clinical guidelines for the pre and post operative physiotherapy management of adults with lower limb amputation (BROOMHEAD et al., 2006) traz que o fisioterapeuta deve fornecer aos pacientes informações sobre locais de reabilitação, estágios do programa e ainda adequar cada indivíduo ao programa de reabilitação de acordo com suas necessidades.

O tema gerador orientação expôs, pelas respostas dos participantes, que a equipe médica e de enfermagem orientaram principalmente em relação à prótese, no pósoperatório imediato. No entanto, orientaram somente a existência de próteses para pessoas amputadas, mas não o processo de aquisição dessa prótese pelo Sistema Único de Saúde (SUS).

Considerando que o ambiente hospitalar é um local onde diversos profissionais da saúde atuam, onde pessoas precisam de cuidados e atenções diárias, entende-se que é papel de todos os profissionais de saúde fornecer orientação não só no momento do pósoperatório imediato, mas sim, visando à alta hospitalar e início da reabilitação no ambiente ambulatorial.

Cabe refletir a comunicação da equipe médica com os indivíduos recém-amputados sobre o processo de reabilitação e, principalmente, quanto à possibilidade da protetização. Entendendo que esta informação é importante, pode-se refletir a tentativa da equipe de empoderar o paciente, suscitar a esperança e amenizar o sofrimento causado pela amputação.

Cientes que nem todas as pessoas amputadas são elegíveis à protetização, além da adequação aos dispositivos de marcha, a protetização pode transcorrer de forma rápida e eficaz, obtendo o indivíduo grande funcionalidade e independência. O contrário pode ser observado através das falhas na reabilitação, que, por sua vez, comprometem o indivíduo. Neste contexto, o processo de aquisição de uma prótese pelo Sistema Único de Saúde (SUS) pode ser lento, demorado, o que dificulta a protetização (SANTOS; LUZ, 2015).

A prótese se tornará, para a pessoa amputada, uma parte do seu corpo, uma 
oportunidade ao retorno às atividades de vida diária (AVDs), trazendo novas possibilidades, por outro lado, pode ser um obstáculo devido ao seu valor elevado e à dificuldade de ser obtida pelo sistema público, diminuindo a esperança de protetização (CHINI; BOEMER, 2007). Shoppen et al. (2001), trazem que a prótese, quando é adequadamente adaptada ao paciente e usada diariamente, é incentivadora, proporcionando condição de melhora da saúde frente à limitação física, favorecendo à reintegração e retorno ao trabalho.

\section{CONSIDERAÇÕES FINAIS}

Pela identificação das percepções dos pacientes, indissociável das práticas dos profissionais no manejo do paciente amputado, evidenciou-se, de certa forma, a aplicabilidade das diretrizes nacionais no ambiente hospitalar. As diretrizes são instrumentos norteadores e servem de guia para os profissionais de saúde, portanto, devem ser mais utilizadas a fim de aperfeiçoar o processo de reabilitação.

A resiliência das pessoas amputadas frente à sua situação e a indagação do que 0 processo de amputação pode acarretar para a pessoa amputada foi abordado no ambiente hospitalar. De uma forma geral, observou-se que os indivíduos não possuíam dúvidas e, assumiram que estas irão surgir com o processo de reabilitação. A protetização também foi abordada pela enfermagem e o psicólogo sendo que este último teve papel importante principalmente, no que concerne o período de manifestação da tristeza pela perda do membro. A família foi identificada como principal responsável pela resiliência do paciente no ambiente hospitalar.

O fisioterapeuta foi apontado como protagonista tanto de execução do processo quanto de orientação e reabilitação. Os enfermeiros e médicos, segundo as falas dos participantes, possuíram dificuldades de estabelecer orientações nos encaminhamentos aos serviços pós-alta e ao processo de reabilitação no nível ambulatorial.

Para que as práticas destacadas nas Diretrizes sejam efetivas, há necessidade de estruturação da rede de cuidados à pessoa com deficiência, organização, investimento e estruturação dos diversos serviços e equipamentos de saúde destinados à reabilitação integral da pessoa amputada, além do diálogo dos profissionais dos diversos níveis de atenção à saúde e massificação quanto à informação desses serviços. Cabe aqui ressaltar, que tanto as diretrizes nacionais quanto as internacionais possuem informações semelhantes quanto ao seu conteúdo no que diz respeito ao cuidado da pessoa amputada. A diretriz norte-americana e a britânica, trazem detalhadamente os processos e fases de reabilitação, focando principalmente para o profissional fisioterapeuta. Já as diretrizes brasileiras seu foco dá-se a equipe multidisciplinar. Como limitações do estudo destaca-se que pela rotina hospitalar e evolução dos pacientes alguns receberam alta em até 48 horas, não sendo possível o deslocamento em tempo para a realização da conversa no leito. 


\section{REFERÊNCIAS}

BRANCO, Ruy Luiz Lorenzetti Branco; DOS SANTOS, Kadine Priscila Bender; DA LUZ, Soraia Cristina Tonon. Promovendo a saúde da pessoa amputada: uma ação educativa chamada conversa no leito/Promoting the health of amputees: an educational action called talking with the bedridden patient. Cadernos Brasileiros de Terapia Ocupacional, São Carlos, v. 25, n. 3, 2017.

BRASIL. Ministério da Saúde. Diretrizes de atenção à pessoa amputada. Departamento de Ações Programáticas Estratégicas. 1. ed. 1. reimp. - Brasília: Ministério da Saúde, 2013a.

http://bvsms.saude.gov.br/bvs/publicacoes/diretrizes_atencao_pessoa_amputada.pdf. Acessado em 06 de junho de 2017.

\section{BRASIL. VIVER SEM LIMITES. Plano Nacional dos Direitos da Pessoa com}

Deficiência. Brasília: 2012. Disponível em: <http://www.brasil.gov.br/viversemlimite/planonacional-dos-direitos-da-pessoa-com-deficiencia>. Acesso em: 22 Mai 2016.

\section{CENSO DEMOGRÁFICO 2010. Características gerais da população, religião e} pessoas com deficiência. Rio de Janeiro: IBGE, 2012. Disponível em: $<$ https://biblioteca.ibge.gov.br/visualizacao/periodicos/94/cd_2010_religiao_deficiencia.pdf >. Acesso em: nov. 2017.

DE OLIVEIRA CHINI, Gislaine Cristina; BOEMER, Magali Roseira. A amputação na percepção de quem a vivencia: um estudo sob a ótica fenomenológica. Revista LatinoAmericana de Enfermagem, Ribeirão Preto, v. 15, n. 2, 2007.

FONTANELLA, Bruno José Bracellos; RICAS, Janete; TURATO, Egberto Ribeiro. Amostragem por saturação em pesquisas qualitativas em saúde: contribuições teóricas. Cadernos de Saúde Pública, Rio de Janeiro, v. 24, n. 1, p. 17-27, Jan. 2008.

GASKELL, George; BAUER, Martin. Pesquisa qualitativa com texto, imagem e som: um manual prático. Petrópolis: Vozes, 2002.

GOVERNO DE SANTA CATARINA. Deliberação 090/CIB/15. Secretaria de Estado da Saúde, Florianópolis, Comissão Intergestores Bipartite. 2015.

HAWKINS, Alexander et al. A systematic review of functional and quality of life assessment after major lower extremity amputation. Annals of vascular surgery, Boston, Massachusetts, v. 28, n. 3, p. 763-780, 2014.

INTERNATIONAL WORKING GROUP ON THE DIABETIC FOOT (IWGDF). Prevention and Management of Foot Problems in Diabetes Guidance Documents and recommendations. Brussels: IWGDF. 2015. Disponível em:

<https://drive.google.com/file/d/OB3SliLeaMnBuR0FYeG9qbWhyeU0/view?pli=1 >. Acesso em: 22 Ago. 2017.

LANGE, Corinna; HEUFT, Gereon. Krankhitsbewältigung und psychotherapie bei patienten nach amputation. Orthopäde, 30, 155-160, 2001.

PETERMANN, Xavéle Braatz et al. Epidemiologia e cuidado à Diabetes Mellitus praticado na Atenção Primária à Saúde: uma revisão narrativa. Revista Saúde, Santa Maria, v. 41, 
n. 1, p. 49-56, 2015.

SAMUEL, Reema et al. Occupational therapy in India: focus on functional recovery and need for empowerment. Indian Journal of Psychiatry, Gurgaon, v. 59, n. 2, p. 242, 2017.

SANTOS, Kadine Priscila; LUZ, Soraia Cristina Tonon. Experiências na Extensão Universitária: Reabilitação de Amputados. Revista brasileira de educação médica, Rio de Janeiro, v. 39, n. 4, p. 602-606, 2015.

SMITH Sara; PURSEY Heather; JONES Amy; BAKER Heidi; SPRINGATE Gemma; RANDELL Tim; MOLONEY Clare; HANCOCK Amanda; NEWCOMBE Lauren; SHAW Carla; ROSE Anna; SLACK Hannah; NORMAN Claire. Clinical guidelines for the pre and post operative physiotherapy management of adults with lower limb amputation. Charted Society of Physiotherapy. London, 2006. Disponível em: < http://bacpar.csp.org.uk/publications/clinical-guidelines-pre-post-operative-physiotherapymanagement-adults-lower-li>. Acess in: 25 Apr 2016.

SOCIEDADE BRASILEIRA DE DIABETES. Diretrizes da Sociedade Brasileira de Diabetes: 2014-2015, São Paulo, 2015. Disponível em:

<http://www.diabetes.org.br/images/2015/area-restrita/diretrizes-sbd-2015.pdf>. Acesso em: 22 Ago. 2017.

THE UNITED STATES DEPARTMENT OF VETERAN AFFAIRS. VA/DoD clinical practice guideline for rehabilitation of lower limb amputation, Washington, D.C, V. 1, n. 1, p. 1-166. 2007. Disponível em:

<http://www.healthquality.va.gov/amputation/amp_sum_correction.pdf>. Acess in: 21 Apr 2015.

VERRONE QUILICI, Maria Teresa et al. Risk factors for foot amputation in patients hospitalized for diabetic foot infection. Journal of diabetes research, Cairo, Egypt, v. 2016, 2016. 